\title{
Reconstruction of physical and biological dose distributions of carbon- ion beam through deconvolution of longitudinal dosimeter responses
}

\author{
N. Kanematsu, T. Inaniwa, S. Yonai, H. Mizuno: National Institute of Radiological \\ Sciences, QST; 4-9-1 Anagawa, Inage-ku, Chiba 263-8555, Japan
}

\section{Purpose}

- Ideal QA dosimetry system for carbon-ion RT

- of highly complex beam fields

- at high spatial and dose resolutions

- especially, for Bragg peaks

- in RBE-weighted (biological) dose

- to evaluate clinical significance of observed deviations.

- This study aims to propose such a system using existing technologies.

\section{Methods}

- Example carbon-ion beam to test:

- Biologically uniform SOBP formed by layer stacking of range-shifted elemental beams (beamlets).

-32 layers at $2.5 \mathrm{~mm}$ range-shift intervals for $8 \mathrm{~cm}$ SOBP

- RCF dosimetry simulation

- RCF response (optical density interpreted as 6MV dose) based on an empirical function of LET.

- Deconvolution of RCF responses

- to estimate weights of beamlets finely arranged at $0.5 \mathrm{~mm}$ range-shift intervals.

- Reconstruction of physical and biological doses

- using a conventional dose-summation algorithm.
Results
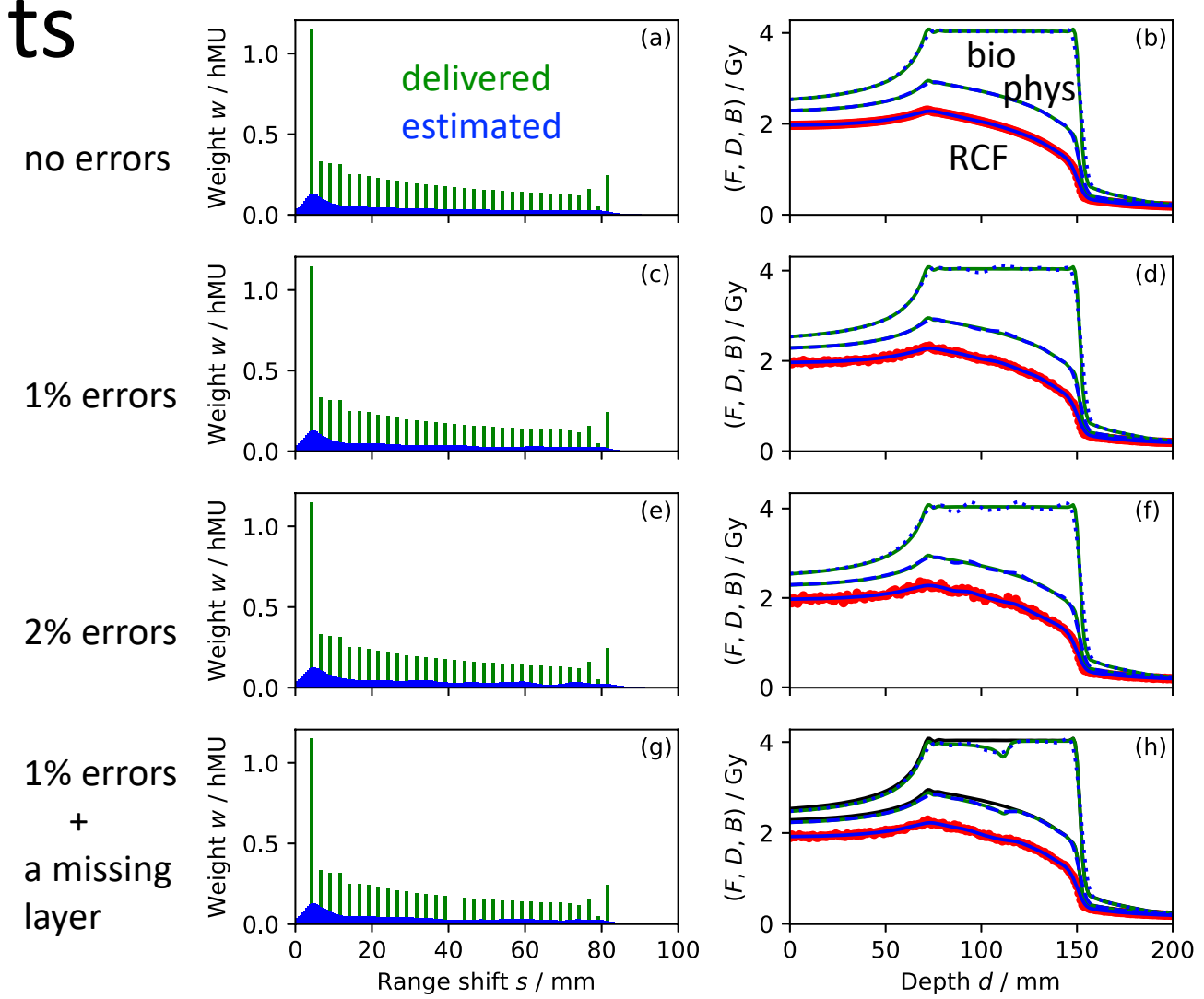

Idea of longitudinal dosimetry with radiochromic film (RCF)

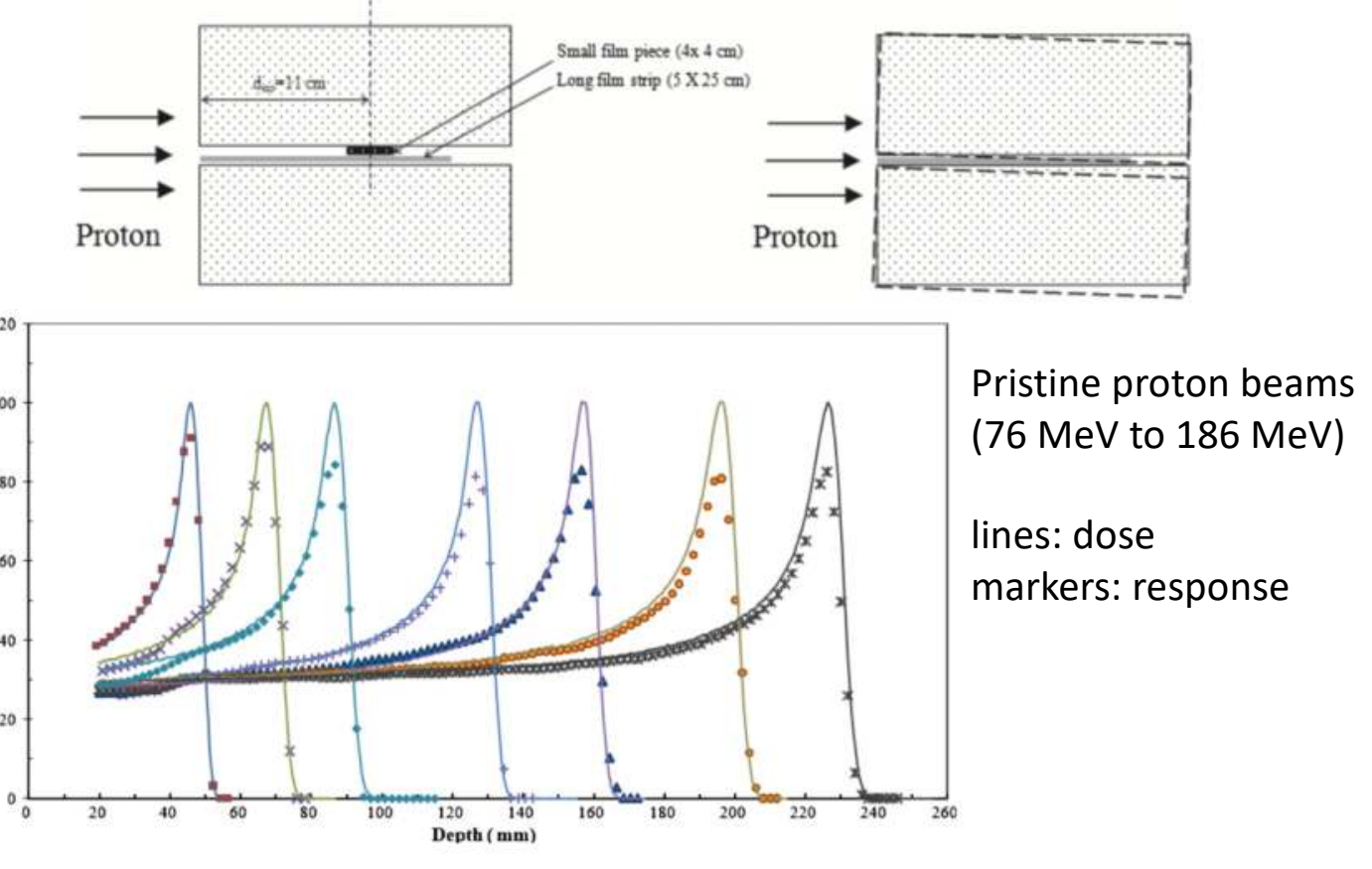

Zao \& Das: Phys Med Biol 55 (2010) N291 (the beamlet without range shifting)

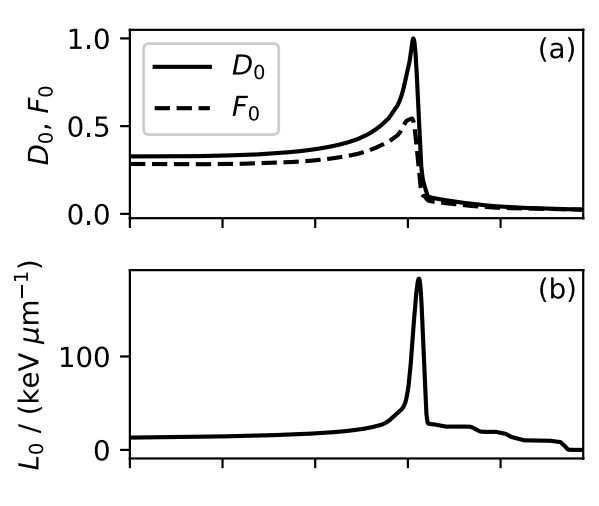

$D_{0}$ : physical dose (measured)

$F_{0}$ : RCF response (to be measured, but

here according to Yonai et al, 2018)

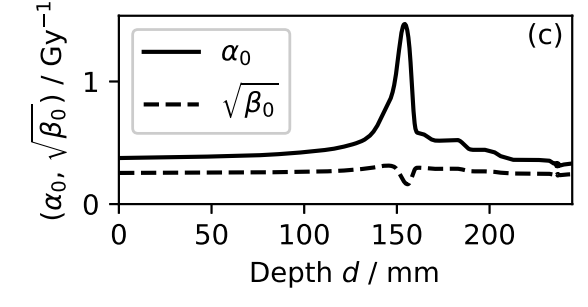

$L_{0}$ : dose-averaged LET (theoretical)

$\alpha_{0}, \beta_{0}$ : LQ radiosensitivity parameters for human salivary gland tumor cells derived from radiobiology experiments

\section{Conclusions}

- This simulation study has shown:

- Physical and biological doses can be measured in longitudinal RCF dosimetry by applying deconvolution algorithm with full beamlet data and RBE model used in treatment planning.

- Dose errors will approximately correspond to RCF measurement errors.

- Beam delivery failure will be easily detectable especially in biological-dose distribution. 